\title{
Genetic Studies for Different Seed Traits in Cucumber (Cucumis sativus L)
}

\author{
Manisha Thakur ${ }^{1 *}$, Ramesh Kumar ${ }^{1}$, Rajesh Kanwar ${ }^{2}$ and D.K. Mehta ${ }^{1}$ \\ ${ }^{1}$ Department of Vegetable Science, ${ }^{2}$ Department of Seed Science and Technology, Dr YS Parmar \\ University of Horticulture and Forestry, Nauni-173 230, Solan, HP, India \\ *Corresponding author
}

\section{A B S T R A C T}

The present investigation was carried out to study the mean performance, heterosis,

\begin{tabular}{|l|}
\hline Ke y w or d s \\
Combining ability, \\
Gene action, \\
Heterosis, Seed \\
germination, Seed \\
vigour index-I and II. \\
\hline Article Info \\
\hline Accepted: \\
28 September 2017 \\
Available Online: \\
10 November 2017 \\
\hline
\end{tabular}
combining ability and gene action for different seed traits viz., seed germination, seed vigour index-I, seed vigour index-II and fruit yield in $15 \mathrm{~F}_{1}$ crosses, developed by crossing 6 genotypes during the year 2015. The seeds of all parents and their crosses, along with standard check (KH-1) were assessed for different seed traits (under laboratory conditions) and fruit yield (under open field conditions) during the year 2016. Experimental results revealed that genotypes PI-618860, Khira-75, UHF-CUC-1 and UHF-CUC-2 were found superior on the basis of mean performance, heterosis and general combining ability studies. The cross combinations viz., Khira-75 x PI-618860, Khira-75 x UHF-CUC-1, Khira-75 x UHF-CUC-2 and Khira-75 x PI-618860, UHF-CUC-1 x PI-618860, UHFCUC-3 x Poinsette, UHF-CUC-3 x PI-618860 were found best on the basis of mean performance, heterosis and specific combining ability studies respectively. Further, Gene action studies indicated the predominant role of non-additive gene action for the control of all the traits under study; hence heterosis breeding can be utilized for the genetic improvement of seed vigour and yield traits in cucumber.

\section{Introduction}

Cucumber (Cucumis sativus L.) is an agriculturally and economically important vegetable crop, ranking fourth in vegetable production worldwide after tomato, cabbage, and onion (FAOSTAT 2016, http://faostat3.fao.org; Xu et al., 2017). Nowadays, it is grown throughout the world in large commercial farms, glasshouses and small gardens. In India, cucumber is cultivated both in summer and rainy seasons, but it cannot withstand cold injury (Rastogi, 1998). It germinates only in an optimal temperature range of 24 to $28{ }^{\circ} \mathrm{C}$. Today, with the emergence of new crop production technologies, demand for high quality vegetable seeds has increased among the farmers. Because, seed is most significant input factor, solely deciding the success or failure of any crop production programme (Bhardwaj and Kumar, 2012). Use of quality seed material is pre-requisite to realizing greater productivity in any crop (Munamava et al., 2004). Further, crop yield also depends on the seed vigor and successful plant establishment under diverse environmental conditions. Moreover, seeds with poor vigor fail to germinate under adverse climatic conditions. So, there is an urgent need to develop new varieties/hybrids of cucumber having greater seed vigor and successful plant 
establishment under different environmental conditions (Finch-Savage and Bassel, 2016).

For developing superior varieties, it is necessary to improve the seed vigour and yield in cucumber. This can be achieved through effective utilization of germplasm resources and integration of genomic tools to impart efficiency and pace of breeding processes (Banga, 2012). Exploitation of heterosis in crop plants is one of the most attractive achievements in boosting up the production and productivity of cucumber. Heterosis breeding can be one of the most viable options for breaking the present yield barrier. Cucumber is monoecious in nature and it gives considerable number of seeds per fruit, which provides an opportunity for the exploitation of hybrid vigour in this crop (Bairagi et al., 2002). Selection of suitable parental line is of utmost importance to exploit heterosis in any crop. Further, the knowledge of combining ability analysis is one of the powerful tools available which give the estimates of combining ability effects and side in selecting desirable parents and cross for further exploitation. Combining ability also specify the nature and magnitude of gene action involved in the expression of different quantitative traits (Machikowa, 2011). The importance of heterosis, combining ability and gene action studies for different yield traits in cucumber have been duly realized earlier by several workers like Mule et al., (2012), Kumar et al., (2013a), Golabadi et al., (2015), Kumar et al., (2016) and Kumar et al., (2017). However, till date inadequate information is available in the literature pertaining to heterosis, combining ability and gene action studies for seed traits in cucumber. Therefore, keeping in view the above facts in mind, we have attempted to improve the seed traits and fruit yield per hectare through different estimates of heterosis, combining ability and gene action in cucumber.

\section{Materials and Methods}

The investigation was carried out at Experimental Research Farm and Laboratory of the Department of Vegetable Science, Dr YS Parmar University of Horticulture and Forestry, Nauni, Solan (HP), India. During the year 2015, crosses were made between 6 genotypes namely Khira-75, UHF-CUC-1, UHF-CUC-2, UHF-CUC-3, Poinsette and PI618860 (Table 1). Simultaneously, each genotype was also selfed to get sufficient seeds for sowing in the next year. Thereafter during the year 2016, seeds of all the parents, $15 \mathrm{~F}_{1}$ hybrids along with standard check cultivar (KH-1) were assessed for different seed traits viz., seed germination (\%), seedling length $(\mathrm{cm})$, seedling dry weight (mg), seed vigor index-I and II under laboratory conditions. Simultaneously, seeds of all the genotypes were also evaluated for fruit yield per hectare (q) in randomized complete block design under open field conditions. During both the years of study, standard cultural practices and plant protection measures for raising a healthy crop stand were adopted as per the recommendations given by Directorate of Extension Education, Dr YS Parmar UHF, Nauni, Solan, HP (Anonymous, 2014).

Seed germination of each genotype was tested under laboratory conditions through blot paper method as per the guidelines of ISTA (Anonymous 1985). The seedling length and seedling dry weight were recorded from 20 randomly selected seedlings of each genotype and averaged to get mean value. Seed vigour index-I and II were calculated as per the formulae given by Abdul-Baki and Anderson (1973). Pickings were made regularly from randomly selected 10 plants and were averaged to have total yield per plant. Then total yield per plant was multiplied with total number of plants accommodating in one hectare to obtain fruit yield per hectare. The 
data recorded for different traits were subjected to analysis of variance in MS Excel2010 worksheet by using the formulae given by Panse and Sukhatme (1967). The additive and dominance components of variance were estimated by following Singh and Chaudhary (1997) and Dabholkar (1992).

The estimates of heterosis over better parent (BP), standard check (SC) were calculated manually in MS Excel-2010 worksheet by using the following formulae given by Singh (1973). Further, statistical significance of different estimates of heterosis was assessed through the t-test formulae adopted by Wynne et al., (1970).

\section{Results and Discussion}

\section{Mean performance and heterosis}

The data recorded for seed germination, seed vigour index-I, seed vigour index-II and fruit yield per hectare showed significant differences among the parents and hybrids (Table 2).

The significant variations were observed among the parents and hybrids for seed germination (parents=80.06-85.53 and hybrids $=80.54-87.79 \%$ ), seed vigor index-I (parents $=2251.00-2799.73$ and hybrids $=2236.78-3014.82$ ), seed vigor indexII (parents $=1182.39$-1338.34 and hybrids=1123.09-1451.41) and fruit yield per hectare (parents $=151.91-290.84$ and hybrids $=119.40-338.25 \quad$ q) (Table 2). Substantial variations for seed germination (Hamid et al., 2002; Kumar et al., 2013b; Kumari, 2015), seed vigour (Nerson, 2007; Kumar et al., 2013b; Kumari, 2015) and yield per hectare (Munshi et al., 2007; Kumar et al., 2008; Hanchinamani et al., 2008; Yadav et al., 2009; Kumar et al., 2010; Singh et al., 2010; Hossain et al., 2010; Dogra and Kanwar, 2011; Kumar et al., 2011a; Golabadi et al., 2012, Singh et al., 2012, Airina et al., 2013, Kumar et al., 2013b, Jat et al., 2015 and Kumar et al., 2017) traits had also been reported earlier in different varieties of cucumber. Among the top five parents and hybrids identified on the basis of mean performance and heterosis studies; it was observed that PI-618860, Khira-75, UHFCUC-1 and UHF-CUC-2 (parents) and Khira75 x PI-618860, Khira-75 x UHF-CUC-1 and Khira-75 x UHF-CUC-2 (hybrids) excelled for most of the traits under study. These cross combinations also resulted in significantly higher positive heterosis over better parent (Table 3) and standard check cultivar (Table 4). These genotypes can be exploited for seed vigour and fruit yield improvement in cucumber.

\section{Combining ability and gene action}

For effective improvement in polygenic traits, information about the combining ability of parents and their crosses, the estimates of genetic components of variance and the type of gene action involved are of prime importance to the breeders.

It guides the breeders to select appropriate parents for heterosis and recombination breeding, hence are important in crop improvement studies. The combining ability studies evaluate the parental lines on the basis of their general combining ability (GCA) effects and the performance of these parents in specific cross combinations (SCA) (Kumar et al., 2016).

Among the parents for GCA effects genotypes, UHF-CUC-1, UHF-CUC-2 and PI-618860 were found good general combiners for seed germination, seed vigour index-I, seed vigour index-II and fruit yield per hectare and exhibited significant positive effects (Table 5). 
Table.1 Sources of cucumber genotypes used as parent and check

\begin{tabular}{ll}
\hline Lines/Parents & Source \\
\hline Khira-75 & Department of Vegetable Science, UHF, Solan, HP \\
UHF-CUC-1 & Department of Vegetable Science, UHF, Solan, HP \\
UHF-CUC-2 & Department of Vegetable Science, UHF, Solan, HP \\
UHF-CUC-3 & Department of Vegetable Science, UHF, Solan, HP \\
Poinsette & IARI, Regional Research Station, Katrain, Kullu Valley, HP \\
PI-618860 & North Central Regional Plant Introduction Station, USA \\
KH-1 (Check) & Department of Vegetable Science, UHF, Solan, HP \\
\hline
\end{tabular}

Table.2 Mean performance of parents, crosses and standard check cultivar for seed traits and fruit yield in cucumber

\begin{tabular}{|c|c|c|c|c|c|}
\hline $\begin{array}{c}\text { Traits } \longrightarrow \\
\text { Parents \& Crosses } \downarrow\end{array}$ & $\begin{array}{c}\text { Seed } \\
\text { Germination }\end{array}$ & $\begin{array}{l}\text { Seed Vigour } \\
\text { Index-I }\end{array}$ & $\begin{array}{l}\text { Seed Vigour } \\
\text { Index-II }\end{array}$ & $\begin{array}{l}\text { Yield Per } \\
\text { Plot (kg) }\end{array}$ & $\begin{array}{c}\text { Yield Per } \\
\text { Hectare (q) }\end{array}$ \\
\hline Khira-75 & $83.40(9.13) * *$ & 2766.70 & 1326.42 & 39.73 & 264.87 \\
\hline UHF-CUC-1 & $84.49(9.19)$ & 2641.21 & 1338.34 & 35.90 & 239.31 \\
\hline UHF-CUC-2 & $83.82(9.16)$ & 2507.08 & 1337.52 & 35.98 & 239.88 \\
\hline UHF-CUC-3 & $80.85(8.99)$ & 2360.86 & 1207.34 & 28.59 & 190.60 \\
\hline Poinsette & $80.06(8.95)$ & 2251.00 & 1182.39 & 22.79 & 151.91 \\
\hline PI-618860 & $85.53(9.25)$ & 2799.73 & 1334.74 & 43.63 & 290.84 \\
\hline Khira-75 x UHF-CUC-1 & $86.46(9.30)$ & 2828.35 & 1368.32 & 48.87 & 325.81 \\
\hline Khira-75 x UHF-CUC-2 & $86.41(9.30)$ & 2869.57 & 1408.86 & 49.06 & 327.04 \\
\hline Khira-75 x UHF-CUC-3 & $81.83(9.05)$ & 2387.14 & 1225.02 & 22.52 & 150.13 \\
\hline Khira-75 x Poinsette & $80.54(8.97)$ & 2236.78 & 1123.09 & 17.91 & 119.40 \\
\hline Khira-75 x PI-618860 & $87.79(9.37)$ & 3014.82 & 1451.41 & 50.74 & 338.25 \\
\hline $\begin{array}{l}\text { UHF-CUC-1 x UHF- } \\
\text { CUC-2 }\end{array}$ & $85.42(9.24)$ & 2631.98 & 1372.41 & 43.84 & 292.25 \\
\hline $\begin{array}{l}\text { UHF-CUC-1 x UHF- } \\
\text { CUC-3 }\end{array}$ & $80.56(8.98)$ & 2239.81 & 1183.51 & 26.01 & 173.38 \\
\hline UHF-CUC-1 x Poinsette & $81.66(9.04)$ & 2644.09 & 1205.92 & 29.28 & 195.17 \\
\hline UHF-CUC-1 x PI-618860 & $85.48(9.25)$ & 2858.31 & 1378.77 & 46.65 & 311.01 \\
\hline $\begin{array}{l}\text { UHF-CUC-2 x UHF- } \\
\text { CUC-3 }\end{array}$ & $81.40(9.02)$ & 2323.89 & 1133.79 & 19.98 & 133.19 \\
\hline UHF-CUC-2 x Poinsette & $82.39(9.08)$ & 2640.47 & 1244.32 & 32.21 & 214.72 \\
\hline UHF-CUC-2 x PI-618860 & $85.58(9.25)$ & 2867.60 & 1439.31 & 46.60 & 310.68 \\
\hline UHF-CUC-3 3 Poinsette & $81.37(9.02)$ & 2341.54 & 1163.12 & 20.99 & 139.96 \\
\hline UHF-CUC-3 x PI-618860 & $81.45(9.03)$ & 2242.18 & 1181.10 & 21.77 & 145.10 \\
\hline Poinsette x PI-618860 & $80.67(8.98)$ & 2334.51 & 1211.23 & 22.59 & 150.58 \\
\hline KH-1 (check) & $82.59(9.09)$ & 2596.32 & 1305.07 & 30.62 & 204.14 \\
\hline Range & 80.54-87.79 & $\begin{array}{l}2236.78- \\
3014.82\end{array}$ & $\begin{array}{l}1123.09- \\
1451.41\end{array}$ & $\begin{array}{l}17.91- \\
50.74\end{array}$ & $\begin{array}{l}119.40- \\
338.25\end{array}$ \\
\hline Population mean & 83.17 & 2562.91 & 1278.27 & 33.47 & 223.10 \\
\hline $\mathrm{SE}(\mathrm{m}) \pm$ & 0.84 & 85.49 & 52.91 & 3.64 & 24.24 \\
\hline $\mathrm{CD}_{(0.05)}$ & 1.68 & $\mathbf{1 7 1 . 1 5}$ & 105.92 & 7.28 & 48.53 \\
\hline
\end{tabular}

*Significant at $5 \%$ level of significance

** Figures present in Parenthesis are square root transformed 
Table.3 Crosses showing heterobeltiosis for seed traits and fruit yield in cucumber

\begin{tabular}{|l|c|c|c|c|c|}
\hline \multicolumn{1}{|c|}{ Traits $\longrightarrow$} & $\begin{array}{c}\text { Seed } \\
\text { Germination }\end{array}$ & $\begin{array}{c}\text { Seed Vigour } \\
\text { Index-I }\end{array}$ & $\begin{array}{c}\text { Seed } \\
\text { Vigour } \\
\text { Index-II }\end{array}$ & $\begin{array}{c}\text { Yield Per } \\
\text { Plot (kg) }\end{array}$ & $\begin{array}{c}\text { Yield Per } \\
\text { Hectare (q) }\end{array}$ \\
\hline Khira-75 x UHF-CUC-1 & $2.33^{*}$ & $2.23^{*}$ & $2.24^{*}$ & $23.01^{*}$ & $23.01^{*}$ \\
\hline Khira-75 x UHF-CUC-2 & $3.09^{*}$ & $3.72^{*}$ & $5.33^{*}$ & $23.48^{*}$ & $23.47^{*}$ \\
\hline Khira-75 x UHF-CUC-3 & -1.88 & $-13.72^{*}$ & $-7.64^{*}$ & $-43.32^{*}$ & $-43.32^{*}$ \\
\hline Khira-75 x Poinsette & $-3.43^{*}$ & $-19.15^{*}$ & $-15.33^{*}$ & $-54.92^{*}$ & $-54.92^{*}$ \\
\hline Khira-75 x PI-618860 & $2.64^{*}$ & $7.68^{*}$ & $8.74^{*}$ & $16.30^{*}$ & $16.30^{*}$ \\
\hline UHF-CUC-1 x UHF- & 1.10 & -0.35 & $2.55^{*}$ & $21.85^{*}$ & $21.83^{*}$ \\
CUC-2 & $-4.65^{*}$ & $-15.20^{*}$ & $-11.57^{*}$ & $-27.55^{*}$ & $-27.55^{*}$ \\
\hline UHF-CUC-1 x UHF- & $-3.35^{*}$ & 0.11 & $-9.89^{*}$ & $-18.44^{*}$ & $-18.44^{*}$ \\
CUC-3 & -0.06 & $2.09^{*}$ & $3.02^{*}$ & $6.92^{*}$ & $6.94^{*}$ \\
\hline UHF-CUC-1 x Poinsette & & & & & \\
\hline UHF-CUC-1 x PI-618860 & $-2.89^{*}$ & $-7.31^{*}$ & $-15.23^{*}$ & $-44.47^{*}$ & $-44.48^{*}$ \\
\hline UHF-CUC-2 x UHF- & -1.71 & $5.32^{*}$ & $-6.97^{*}$ & $-10.48^{*}$ & $-10.49^{*}$ \\
CUC-3 & -1.50 & $2.42^{*}$ & $7.61^{*}$ & $6.81^{*}$ & $6.82^{*}$ \\
\hline UHF-CUC-2 x Poinsette & -0.82 & $-3.66^{*}$ & $-26.58^{*}$ & $-26.57^{*}$ \\
\hline UHF-CUC-2 x PI-618860 & $-4.77^{*}$ & $-19.91^{*}$ & $-11.51^{*}$ & $-50.10^{*}$ & $-50.11^{*}$ \\
\hline UHF-CUC-3 x Poinsette & $-5.68^{*}$ & $-16.62^{*}$ & $-9.25^{*}$ & $-48.22^{*}$ & $-48.23^{*}$ \\
\hline UHF-CUC-3 x PI-618860 & $\mathbf{- 5 . 6 8 - 3 . 0 9}$ & $\mathbf{- 1 9 . 9 1 - 7 . 6 8}$ & $\mathbf{- 1 5 . 3 3 - 8 . 7 4}$ & $\mathbf{- 5 4 . 9 2 - 2 3 . 4 8}$ & $\mathbf{- 5 4 . 9 2 - 2 3 . 4 7}$ \\
\hline Poinsette x PI-618860 & & &
\end{tabular}

Table.4 Crosses showing standard heterosis for seed traits and fruit yield in cucumber

\begin{tabular}{|l|c|c|c|c|c|}
\hline \multicolumn{1}{|c|}{ Traits } & $\begin{array}{c}\text { Seed } \\
\text { Germination }\end{array}$ & $\begin{array}{c}\text { Seed Vigour } \\
\text { Index-I }\end{array}$ & $\begin{array}{c}\text { Seed Vigour } \\
\text { Index-II }\end{array}$ & $\begin{array}{c}\text { Yield Per } \\
\text { Plot (kg) }\end{array}$ & $\begin{array}{c}\text { Yield Per } \\
\text { Hectare (q) }\end{array}$ \\
\hline Khira-75 x UHF-CUC-1 & $4.69^{*}$ & $8.94^{*}$ & $4.85^{*}$ & $59.60^{*}$ & $59.60^{*}$ \\
\hline Khira-75 x UHF-CUC-2 & $4.63^{*}$ & $10.52^{*}$ & $7.95^{*}$ & $60.22^{*}$ & $60.20^{*}$ \\
\hline Khira-75 x UHF-CUC-3 & -0.92 & $-8.06^{*}$ & $-6.13^{*}$ & $-26.45^{*}$ & $-26.46^{*}$ \\
\hline Khira-75 x Poinsette & $-2.48^{*}$ & $-13.85^{*}$ & $-13.94^{*}$ & $-41.51^{*}$ & $-41.51^{*}$ \\
\hline Khira-75 x PI-618860 & $6.30^{*}$ & $16.12^{*}$ & $11.21^{*}$ & $65.71^{*}$ & $65.70^{*}$ \\
\hline $\begin{array}{l}\text { UHF-CUC-1 x UHF- } \\
\text { CUC-2 }\end{array}$ & $3.43^{*}$ & 1.37 & $5.16^{*}$ & $43.17^{*}$ & $43.16^{*}$ \\
\hline $\begin{array}{l}\text { UHF-CUC-1 x UHF- } \\
\text { CUC-3 }\end{array}$ & $-2.46^{*}$ & $-13.73^{*}$ & $-9.31^{*}$ & $-15.06^{*}$ & $-15.07^{*}$ \\
\hline UHF-CUC-1 x Poinsette & -1.13 & 1.84 & $-7.60^{*}$ & $-4.38^{*}$ & $-4.39^{*}$ \\
\hline UHF-CUC-1 x PI-618860 & $3.50^{*}$ & $10.09^{*}$ & $5.65^{*}$ & $52.35^{*}$ & $52.35^{*}$ \\
\hline $\begin{array}{l}\text { UHF-CUC-2 x UHF- } \\
\text { CUC-3 }\end{array}$ & -1.44 & $-10.49^{*}$ & $-13.12^{*}$ & $-34.75^{*}$ & $-34.76^{*}$ \\
\hline UHF-CUC-2 x Poinsette & -0.24 & 1.70 & $-4.65^{*}$ & $5.19^{*}$ & $5.18^{*}$ \\
\hline UHF-CUC-2 x PI-618860 & 2.01 & $10.45^{*}$ & $10.29^{*}$ & $52.19^{*}$ & $52.19^{*}$ \\
\hline UHF-CUC-3 x Poinsette & -1.48 & $-9.81^{*}$ & $-10.88^{*}$ & $-31.45^{*}$ & $-31.44^{*}$ \\
\hline UHF-CUC-3 x PI-618860 & -1.38 & $-13.64^{*}$ & $-9.50^{*}$ & $-28.90^{*}$ & $-28.92^{*}$ \\
\hline Poinsette x PI-618860 & $-2.32^{*}$ & $-10.08^{*}$ & $-7.19^{*}$ & $-26.22^{*}$ & $-26.24^{*}$ \\
\hline Range & $\mathbf{- 2 . 4 8 - 6 . 3 0}$ & $\mathbf{- 1 3 . 8 5 - 1 6 . 1 2}$ & $\mathbf{- 1 3 . 9 4 - 1 1 . 2 1}$ & $\mathbf{- 4 1 . 5 1 - 6 5 . 7 1}$ & $\mathbf{- 4 1 . 5 1 - 6 5 . 7 0}$ \\
\hline
\end{tabular}


Table.5 Estimates of general combining ability effects of parents for different seed traits and fruit yield in cucumber

\begin{tabular}{lccccc}
\hline $\begin{array}{c}\text { Traits } \\
\text { Parents } \downarrow\end{array}$ & $\begin{array}{c}\text { Seed } \\
\text { germination } \\
(\boldsymbol{\%})\end{array}$ & $\begin{array}{c}\text { Seed vigour } \\
\text { Index I }\end{array}$ & $\begin{array}{c}\text { Seed vigour } \\
\text { Index II }\end{array}$ & $\begin{array}{c}\text { Marketable yield } \\
\text { per plot (kg) }\end{array}$ & $\begin{array}{c}\text { Marketable yield } \\
\text { per hectare }(\mathbf{q})\end{array}$ \\
\hline Khira-75 & -0.11 & 20.51 & 14.27 & 1.39 & 9.28 \\
UHF-CUC-1 & $1.45^{*}$ & $109.63^{*}$ & $43.05^{*}$ & $4.83^{*}$ & $32.22^{*}$ \\
UHF-CUC-2 & $0.80^{*}$ & $56.70^{*}$ & $40.05^{*}$ & $4.93 *$ & $32.87^{*}$ \\
UHF-CUC-3 & $-1.05^{*}$ & $-98.19^{*}$ & $-46.49^{*}$ & $-4.92^{*}$ & $-32.77^{*}$ \\
Poinsette & $-1.69^{*}$ & $-153.95^{*}$ & $-84.49^{*}$ & $-8.66^{*}$ & $-57.73^{*}$ \\
PI-618860 & $0.60^{*}$ & $65.31^{*}$ & $33.60^{*}$ & $2.42^{*}$ & $16.13^{*}$ \\
\hline SE(gi) & 0.191 & 19.689 & 11.984 & 0.84 & 5.597 \\
SE (gi-gj) & 0.295 & 30.502 & 18.566 & 1.301 & 8.671 \\
CD (gi) & $\mathbf{0 . 4 0}$ & $\mathbf{4 0 . 9 5}$ & $\mathbf{2 4 . 9 3}$ & $\mathbf{1 . 7 5}$ & $\mathbf{1 1 . 6 4}$ \\
CD (gi-gj) & 0.61 & 63.44 & 38.62 & 2.71 & 18.04 \\
\hline
\end{tabular}

*Significant at $5 \%$ level of significance

Table.6 Estimates of specific combining effects of parents for different seed traits and fruit yield in cucumber

\begin{tabular}{|c|c|c|c|c|c|}
\hline $\begin{array}{l}\text { Traits } \\
\text { Parents }\end{array}$ & $\begin{array}{c}\text { Seed } \\
\text { germination } \\
(\%)\end{array}$ & $\begin{array}{l}\text { Seed vigour } \\
\text { Index-I }\end{array}$ & $\begin{array}{c}\text { Seed } \\
\text { vigour } \\
\text { Index-II } \\
\end{array}$ & $\begin{array}{c}\text { Yield per plot } \\
(\mathrm{Kg})\end{array}$ & $\begin{array}{c}\text { Yield per } \\
\text { hectare }(q)\end{array}$ \\
\hline Khira-75 x UHF-CUC-1 & -0.05 & -50.24 & 4.02 & -3.93 & -26.19 \\
\hline Khira-75 x UHF-CUC-2 & -0.07 & $-131.45^{*}$ & 6.20 & -3.94 & -26.28 \\
\hline Khira-75 x UHF-CUC-3 & $-1.19 *$ & $-122.77 *$ & -37.44 & -1.49 & -9.92 \\
\hline Khira-75 x Poinsette & $-1.34 *$ & $-176.88 *$ & -24.39 & -3.55 & -23.65 \\
\hline Khira-75 x PI-618860 & $1.84^{*}$ & $152.60 *$ & 9.87 & $6.21 *$ & $41.43^{*}$ \\
\hline UHF-CUC-1 x UHF-CUC-2 & 0.97 & $141.93 *$ & 48.76 & $5.69 *$ & $37.96 *$ \\
\hline UHF-CUC-1 x UHF-CUC-3 & $-1.77 *$ & $-185.61 *$ & -48.54 & $-11.00 *$ & $-73.33 *$ \\
\hline UHF-CUC-1 x Poinsette & $-2.42 *$ & $-280.22 *$ & $-112.47 *$ & -11.86 & $-79.09 *$ \\
\hline UHF-CUC-1 x PI-618860 & $2.55^{*}$ & $278.57 *$ & $97.77 *$ & $9.88^{*}$ & $-65.90 *$ \\
\hline UHF-CUC-2 x UHF-CUC-3 & $-2.39 *$ & $-280.02 *$ & $-87.05^{*}$ & $-7.61 *$ & $-50.73 *$ \\
\hline UHF-CUC-2 x Poinsette & -0.65 & $180.03 *$ & -26.64 & -0.60 & $-3.98 *$ \\
\hline UHF-CUC-2 x PI-618860 & 0.89 & $174.98 *$ & 28.12 & $5.70^{*}$ & $38.01 *$ \\
\hline UHF-CUC-3 x Poinsette & $1.93 *$ & $331.30^{*}$ & $98.30^{*}$ & $12.18 *$ & $81.21 *$ \\
\hline UHF-CUC-3 x PI-618860 & $2.83^{*}$ & $339.17 *$ & $175.20 *$ & $15.50 *$ & $103.32 *$ \\
\hline Poinsette x PI-618860 & -0.65 & $-230.50 *$ & -45.01 & $-5.60 *$ & $-37.30 *$ \\
\hline SE(sij) & 0.524 & 54.075 & 32.913 & 2.306 & 15.373 \\
\hline SE(sij-sik) & 0.10 & 0.10 & 0.10 & 0.10 & 0.10 \\
\hline SE(sij-skl) & 0.724 & 74.715 & 45.476 & 3.186 & 21.24 \\
\hline CD(sij) & 1.09 & 112.48 & 68.46 & 4.80 & 31.98 \\
\hline CD(sij-sik) & 0.21 & 0.21 & 0.21 & 0.21 & 0.21 \\
\hline CD(sij-skl) & 1.51 & 155.41 & 94.59 & 6.63 & 44.18 \\
\hline
\end{tabular}


Table.7 Estimates of genetic components of variance for different seed traits and fruit yield in cucumber

\begin{tabular}{|l|c|c|c|c|c|c|}
\hline Character & gca & sca & $\boldsymbol{\sigma}^{2} \mathbf{g}$ & $\boldsymbol{\sigma}^{2} \mathbf{s}$ & $\begin{array}{c}\text { Variance ratio } \\
\left(\boldsymbol{\sigma}^{2} \mathbf{g} / \mathbf{\sigma}^{\mathbf{2}} \mathbf{s}\right)\end{array}$ & $\begin{array}{c}\text { Predictability ratio } \\
\left(\mathbf{2 \sigma ^ { 2 }} \mathbf{g} / \mathbf{2} \boldsymbol{\sigma}^{2} \mathbf{g}+\boldsymbol{\sigma}^{2} \mathbf{s}\right)\end{array}$ \\
\hline Seed germination (\%) & 11.307 & 3.946 & 1.28 & 2.90 & 0.44 & 0.46 \\
\hline Seed vigour Index-I & 85212.818 & 59190.534 & 9256.02 & 48025.86 & 0.19 & 0.27 \\
\hline Seed vigour Index-II & 22542.765 & 6966.154 & 2300.82 & 2829.97 & 0.81 & 0.62 \\
\hline Marketable yield per plot (kg) & 247.309 & 87.835 & 28.38 & 67.53 & 0.42 & 0.45 \\
\hline Marketable yield per hectare (kg) & 10992.199 & 3903.782 & 1261.24 & 3001.48 & 0.42 & \multicolumn{2}{c|}{0.45} \\
\hline
\end{tabular}

Similar findings have been reported by Kumar, 2013 and Kumari, 2015 for seed germination, seed vigour index-I and seed vigour index-II and Kumbhar et al., (2005), Munshi et al., (2006), Singh and Sharma (2006), Yadav et al., (2007), Hanchinamani and Patil (2009), Dogra and Kanwar (2011), Khuswaha et al., (2011), Kumar et al., (2011b), Olfati et al., (2012), Bairagi et al., (2013), Kumar et al., (2013), Golabadi et al., (2015) and Kumar et al., (2017) for fruit yield in cucumber.

For various seed traits viz., seed germination, seed vigour index-I, seed vigour index-II and fruit yield, crosses Khira-75 x PI-618860, UHF-CUC-1 x PI-618860, UHF-CUC-3 x Poinsette and UHF-CUC-3 x PI-618860 recorded significantly high positive specific combining ability (SCA) effects among all the hybrids under study (Table 6). Similar findings for seed traits have been reported by Kumar, 2013 and Kumari, 2015. These crosses indicated the role of both additive and non-additive gene action for improvement of these seed traits. The crosses having the parents with good $\times$ good GCA effects can be utilized to get transgressive segregants in early generations i.e., $\mathrm{F}_{2}$. While, hybrid combination having the parents with good $\times$ average GCA effects may give desirable transgressive segregants in the later segregating generations and cross combinations having the parents with good $x$ poor GCA effects may be utilized for exploitation of heterosis in $F_{1}$ generation.
However, for fruit yield Dogra and Kanwar (2011), Kushwaha et al., (2011), Kumar et al., (2013a), Golabadi et al., (2015), Kalidas et al., (2015) and Kumar et al., (2017) had also reported significant positive GCA and SCA effects.

A perusal of the data presented in table 7 indicated that the estimates of $\sigma^{2} \mathrm{SCA}$ were higher in magnitude as compared to $\sigma^{2}$ GCA (average) for all the traits under study, thereby indicating the predominant role of non-additive gene action governing all the traits. Further, predictability ratio $\left(2 \sigma^{2} g /\left(2 \sigma^{2} g+\sigma^{2} s\right)\right.$ was found less than one for all the traits viz., seed germination (0.46), seed vigour index-I (0.27), seed vigour indexII (0.62) and fruit yield per hectare (0.45). Again it confirmed the role of non-additive gene action controlling all the traits under study; hence heterosis breeding can be utilized for the improvement of seed vigour and yield traits in cucumber. Dogra and Kanwar (2011), Kumar et al., (2013a), Golabadi et al., (2015) and Kumar et al., (2017) had also reported pre-dominance of non-additive gene action for fruit yield in cucumber.

From the present studies it is concluded that genotypes Khira-75, UHF-CUC-1, UHFCUC-2 and PI-618860 were found superior on the basis of mean performance and general combining ability studies. While cross combination viz., Khira-75× PI-618860, Khira-75× UHF-CUC-1, Khira-75× UHF- 
CUC-2 and Khira-75 x PI-618860, UHFCUC-1 x PI-618860, UHF-CUC-3 x Poinsette, UHF-CUC-3 x PI-618860 were found best on the basis of mean performance, heterosis and specific combining ability studies respectively. These, hybrids can be released for commercial cultivation in different parts of the country after multilocation testing. While identified novel genotypes viz., Khira-75, UHF-CUC-1, UHFCUC-2 and PI-618860 can be utilized worldwide for the exploitation of heterosis for seed vigour and yield improvement in cucumber.

\section{Acknowledgement}

I am very grateful to the Department of Science and Technology for providing INSPIRE Fellowship to my $\mathrm{Ph}$.D programme and also thankful to the North Central Regional Plant Introduction Station, USA and ICAR-Indian Agricultural Research Institute (IARI) Regional Station, Katrain, Kullu Valley, HP, India for providing cucumber germplasm to carry out present investigation.

\section{References}

Abdul-Baki, A.A. and Anderson, J.D. 1973. Vigor germinated in soybean seed by multiple criteria. Crop Science 13: 630633.

Airina, C.K., Pradeepkumar, T., George, T.E., Sadhankumar, P.G. and Krishnan, S. 2013. Heterosis breeding exploiting gynoecy in cucumber (Cucumis sativus L.). Journal of Tropical Agriculture51 (1-2): 144-148.

Allard, R.W. 1960. Principles of plant Breeding. John Wiley and Sons. Inc., U.S.A. p. 485 .

Anonymous. 1985. International rules for seed testing. Seed Science Technology13: 293-353.
Anonymous. 2014. Package of practices for vegetable crops. Solan: Dr. Y.S. Parmar University of Horticulture and Forestry, Nauni, Solan, HP, India. 202p.

Bairagi, S.K., Ram, H.H. and Singh, D.K. 2013. Analysis of combining ability in cucumber (Cucumis sativus L.) through half diallel mating system. Annals of Horticulture 6 (2): 308-314.

Bairagi, S.K., Singh, D.K. and Ram, H.H. 2002. Studies on heterosis for yield attributes in cucumber (Cucumis sativus L.). Vegetable Science 29:75-77.

Banga, S.S. 2012. Germplasm Enhancement in Indian Mustard: Some Exiting New Developments. In: "Souvenir of XIX Annual AICRP Group Meet on Rapeseed-Mustard", Birsa Agricultural University, Ranchi, India, PP. 29-34.

Bhardwaj, R.K. and Kumar, S. 2012. Studies on correlation between yield and seed characters in cucumber (Cucumis sativus L.). International journal of Farm Sciences2 (1): 54-58.

Dabholkar, A.R. 1992. Elements of biometrical genetics. New Delhi: Concept Publishing Company. pp. 187214.

Dogra, B.S. and Kanwar, M.S. 2011. Exploitation of combining ability in cucumber (Cucumis sativus L.). Research Journal of Agricultural sciences2 (1): 55-59.

Finch-Savage, W.E. and Bassel, G.W. 2016. Seed vigour and crop establishment: extending performance beyond adaptation. Journal of Experimental Botany67 (3): 567-591.

Goggi, A.S., Caragea, P., Pollak, L., Mcandrews, G., Devries, M. and Montgomer, Y.K. 2008. Seed quality assurance in maize breeding programs: tests to explain variations in maize inbreds and populations. Agronomy Journal100 (2): 343-337.

Golabadi, M., Golkar, P. and Eghtedary, A. 
2015. Combining ability analysis of fruit yield and morphological traits in greenhouse cucumber (Cucumis sativus L.). Canadian Journal of Plant Science 95(2): 377-385.

Golabadi, M., Golkar, P. and Eghtedary, A.R. 2012. Assessment of genetic variation in cucumber (Cucumis sativus L.) genotypes. European Journal of Experimental Biology2 (3): 826-831.

Hamid, A., Baloch, J.U. and Khan, N. 2002. Performance of six cucumber (Cucumis sativus L.) genotypes in Swat-Pakistan. International Journal of Agriculture and Biology 4 (4): 491-492.

Hanchinamani, C.N. and Patil, M.G. 2009. Combining ability through line $\mathrm{x}$ tester analysis in cucumber (Cucumis sativus L.). Asian Journal of Horticulture 4 (1): 70-73.

Hanchinamani, C.N., Patil, M.G., Dharmatti, P.R. and Mokashi, A.N. 2008. Studies on variability in cucumber (Cucumis sativus L.). Crop Research 36 (1\&3): 273-276.

Hossain, M.F., Rabbani, M.G., Hakim, M.A., Amanullah, A.S.M. and Ahsanullah, A.S.M. 2010. Studies on variability, character association and yield performance of cucumber (Cucumis sativus L.) Bangladesh Research Publications Journal 43: 297-311.

Jat, G.S., Munshi, A.D., Behera, T.K., Choudhary, H. and Dev, B. 2015. Exploitation of heterosis in cucumber for earliness, yield and yield components utilizing gynoecious lines. Indian Journal of Horticulture 72(4): 494-499.

Kalidas, P., Munshi, A.D., Behera, T.K., Kumar, R. and Karmakar, P. 2015. Estimating combining ability for yield and yield contributing traits in cucumber. Indian Journal of Horticulture 72 (1): 49-53.

Kempthorne, O. 1957.An introduction to genetic statistics. NewYork: John Wiley and Sons. pp. 458-471.

Khushwaha, M., Yadav, L.B. and Maurya, R.P. 2011. Heterobeltiosis and combining ability in cucumber (Cucumis sativus L.) under mid hilly area of Uttrakhand. Progressive Agriulture 11(1): 103-107.

Kumar, A., Kumar, S. and Pal, A.K. 2008. Genetic variability and characters association for fruit yield and yield traits in cucumber. Indian Journal of Horticulture 65 (4): 423-428.

Kumar, J., Munshi, A.D., Kumar, R., Sureja, A.K. and Sharma, R.K. 2011b. Combining ability and its relationship with gene action in slicing cucumber. Indian Journal of Horticulture 68 (4): 507-511.

Kumar, J., Munshi, A.D., Kumar, R., Sureja, A.K. and Sharma, R.K. 2013a. Combining ability and its relationship with gene action in slicing cucumber. Indian Journal of Horticulture 70 (1): 135-138.

Kumar, K.H.A., Patil, G., Hanchinamani, N., Goud, S. and Hiremath, V.K. 2011a. Genetic relationship of growth and development traits with fruit yield in $\mathrm{F}_{2}$ population of BGDL $x$ Hot season of cucumber (Cucumis sativus L.). Journal of Agricultural Sciences 24 (4): 497500.

Kumar, S. 2010. Genetic evaluation of cucumber (Cucumis sativus L.) germplasm for yield and quality traits. MSc Thesis, Dr YS Parmar University of Horticulture and Forestry, Nauni, Solan (HP). 68p.

Kumar, S. 2013. Genetic studies on yield and quality traits in cucumber (Cucumis sativus L.). PhD Thesis, Dr YS Parmar University of Horticulture and Forestry, Nauni, Solan (HP). 153p.

Kumar, S., Kumar, D., Kumar, R., Thakur, K.S. and Dogra, B.S. 2013b. Estimation 
of genetic variability and divergence for fruit yield and quality traits in cucumber (Cucumis sativus L.) in North-Western Himalayas. Universal Journal of Plant Science 1:27-36.

Kumar, S., Kumar, R., Kumar, D., Gautam, N., Dogra, R.K., Mehta, D.K., Sharma, H.D. and Kansal, S. 2016.Parthenocarpicgynoecious parental lines of cucumber introduced from Netherlands for developing highyielding, quality hybrids. Journal of Crop Improvement30: 352-369.

Kumar, S., Kumar, R., Kumar, D., Gautam, N., Singh, N., Parkash, C., Dhiman, M.R. and Shukla, Y.R. 2017. Heterotic potential, potence ratio, combining ability and genetic control of yield and its contributing traits in cucumber (Cucumis sativus L.). New Zealand Journal of Crop and Horticultural Science45 (1): 1-16.

Kumari, R. 2015. Studies on residual heterosis and combining ability in cucumber (Cucumis sativus L.) MSc Thesis, Dr YS Parmar University of Horticulture and Forestry, Nauni, Solan (HP). 135p.

Kumbhar, H.C., Dumbre, A.D. and Patil, H.E. 2005. Heterosis and combining ability studies in cucumber (Cucumis sativus L.). Journal of Maharashtra Agricultural Universities 30 (3): 272275.

Kushwaha, M., Yadav, L.B. and Maurya, R.P. 2011. Heterobeltiosis and combining ability in cucumber (Cucumis sativus L.) under mid hilly area of Uttrakhand. Progressive Agriculture 11:103-107.

Machikowa, T., Saetang, C. and Funpeng, K. 2011. General and specific combining ability for quantitative characters in sunflower. Journal of Agricultural Science3 (1): 91-95.

Mule, P.N., Khandelwal, V., Lodam, V.A., Shinde, D.A., Patil, P.P. and Patil, A.B.
2012. Heterosis and combining ability in cucumber (Cucumis sativus L.). Madras Agricultural Journal 99 (7/9): 420-423.

Munamava, M.R., Goggi, A. S. and Pollak, L. 2004. Seed quality of maize inbred lines with different composition and genetic backgrounds. Crop Science44: 542-548.

Munshi, A.D., Kumar, R. and Panda, B. 2006. Combining ability in cucumber (Cucumis sativus L.). Indian Journal of Agricultural Sciences 76 (12): 750-752.

Munshi, A.D., Panda, B., Behera, T.K., Kumar, R., Bisht, I.S. and Behera, T.K. 2007. Genetic variability in Cucumis sativus var. hardwickii R. germplasm. Cucurbit Genetics Cooperative 30: 510.

Nerson, H. 2007. Seed production and germinability of cucurbit crops. Seed Science and Biotechnology1 (1): 1-10.

Olfati, J.A., Samizadeh, H., Rabiei, B. and Peyvast, G.H. 2012. Griffing's methods comparison for general and specific combining ability in cucumber. Scientific World Journal1: 1-4.

Panse, V.G. and Sukhatme, P.V. 1967. Statistical methods for agricultural workers. New Delhi: Indian Council of Agricultural Research. 381p.

Rastogi, K.B. 1998. Cucumber hybrid production. In: Breeding and Seed Production of vegetable crops by Centre of Advanced Studies in Horticulture (Vegetable Science). Department of Vegetable Science, UHF, Nauni, Solan. pp.76-80.

Singh, D. 1973.Diallel analysis over environments-I. Indian Journal of Genetics and Plant Breeding 33: 127136.

Singh, R., Singh, A.K., Kumar, S. and Singh, B.K. 2012. Heterosis and inbreeding depression for fruit characters in cucumber. Indian Journal of Horticulture69 (2): 200-204. 
Singh, R.K. and Chaudhary, B.D. 1997. Biometrical Methods in Quantitative Genetic analysis. Ludhiana: Kalyani Publishers. 342p.

Singh, S.K., Kishor, G.R. and Srivastava, J.P. 2010. Commercial exploitation of hybrid vigour in cucumber. Progressive Agriculture 10: 266-269.

Singh, Y. and Sharma, S. 2006. Combining ability through line $\mathrm{x}$ tester analysis in cucumber (Cucumis sativus L.). Crop Research, Hissar 31(1): 110-115.

Wynne, J.C., Emery, D.A. and Rice, P.M. 1970. Combining ability estimates in Arachishypogeae L. II. Field performance of $\mathrm{F}_{1}$ hybrids. Crop Science 10:713-715.

Xu, X., Ji, J., Xu, Q., Qi, X. and Chen,
X.2017. Inheritance and quantitative trail loci mapping of adventitious root numbers in cucumber seedlings under waterlogging conditions. Mol Genet Genomics 292: 353-364

Yadav, J.R., Singh, S.P., Prihar, N.S., Yadav, J.K., Mishra, G., Kumar, S. and Yadav, A. 2007. Combining ability for yield and its contributing characters in cucumber (Cucumis sativus L.). Progressive Agriculture 7 (1\&2): 116118.

Yadav, Y.C., Kumar, S., Brijpal, B. and Dixit, S.K. 2009. Genetic variability, heritability and genetic advance for some traits in cucumber. Indian Journal of Horticulture 66 (4): 488-491.

\section{How to cite this article:}

Manisha Thakur, Ramesh Kumar, Rajesh Kanwar and Mehta, D.K. 2017. Genetic Studies for Different Seed Traits in Cucumber (Cucumis sativus L). Int.J.Curr.Microbiol.App.Sci. 6(11): 3939-3949. doi: https://doi.org/10.20546/ijcmas.2017.611.460 